\title{
Oscillations for Nonlinear Neutral Delay Differential Equations with Variable Coefficients
}

\author{
Fatima N. Ahmed, Rokiah R. Ahmad, Ummul K. S. Din, and Mohd S. M. Noorani
}

School of Mathematical Sciences, Faculty of Science and Technology, Universiti Kebangsaan Malaysia, 43600 Bangi, Selangoor, Malaysia

Correspondence should be addressed to Fatima N. Ahmed; zahra80zahra@yahoo.com

Received 11 July 2014; Accepted 18 August 2014; Published 14 October 2014

Academic Editor: Allan Peterson

Copyright (C) 2014 Fatima N. Ahmed et al. This is an open access article distributed under the Creative Commons Attribution License, which permits unrestricted use, distribution, and reproduction in any medium, provided the original work is properly cited.

A class of nonlinear neutral delay differential equations is considered. Some new oscillation criteria of all solutions are derived. The obtained results generalize and extend some of well known previous results in the literature.

\section{Introduction and Preliminaries}

Consider the nonlinear neutral delay differential equation of the form

$$
\begin{aligned}
& (a(t) x(t)-p(t) x(t-\tau))^{\prime} \\
& \quad+q(t) \prod_{i=1}^{n}\left|x\left(t-\sigma_{i}\right)\right|^{\alpha_{i}} \operatorname{sign} x\left(t-\sigma_{i}\right)=0,
\end{aligned}
$$

where

$$
\begin{array}{r}
a(t), p(t), q(t) \in C\left(\left[t_{0}, \infty\right), \mathbb{R}^{+}\right), \quad \tau, \sigma_{i}>0, \\
\alpha_{i} \geq 0, \quad \sum_{i=1}^{n} \alpha_{i}=1, \\
i=1,2, \ldots, n .
\end{array}
$$

Let $m=\max \left\{\tau, \sigma_{i}, 1 \leq i \leq n\right\}$. By a solution of (E) we mean a function $x \in C[[\tilde{t}-m, \infty), \mathbb{R}]$ for some $\tilde{t} \geq t_{0}$ such that $a(t) x(t)-p(t) x(t-\tau)$ is continuously differentiable for $t \geq \tilde{t}$ and such that (E) is satisfied for $t \geq \tilde{t}$. Let $\tilde{t} \geq t_{0}$ be a given initial point and let $\Phi \in C[[\widetilde{t}-m, \widetilde{t}], \mathbb{R}]$ be a given initial function. Then, one can show by using the method of steps that $(\mathrm{E})$ has a unique solution on $[\tilde{t}, \infty)$ satisfying the initial function

$$
x(t)=\Phi(t), \quad \tilde{t}-m \leq t \leq \tilde{t} .
$$

As usual, a solution of (E) is said to be oscillatory if it has arbitrarily large zeros and nonoscillatory if it is either eventually positive or eventually negative. Equation $(\mathrm{E})$ is said to be oscillatory if all its solutions are oscillatory.

In the sequel, unless otherwise specified, when we write a functional inequality, we assume that it holds for all sufficiently large $t$.

In the case where $n=1, a(t) \equiv 1$, and $p$ and $q$ are constants, Karpuz and Öcalan [1] improved the result of Ladas and Sficas [2] holding $0 \leq p \leq 1, q \geq 0$, and $q(\tau-\sigma)>$ $(1 / e)(1-p)$ conditions for oscillation. Also, the case including continuous functions as coefficients

$$
(x(t)-p(t) x(t-\tau))^{\prime}+q(t) x(t-\sigma)=0, \quad t \geq t_{0},
$$

has been studied by many authors; see, for example, Kubiaczyk and Saker [3], Karpuz and Öcalan [1], Ahmed at al. [4], Chuanxi et al. [5], and Yu et al. [6]. In particular, Chen et al. [7] succeeded in getting some oscillation theorems for (3) which involve joint behaviour of $p$ and $q$ using the condition

$$
p(t-\sigma) q(t) \leq q(t-\tau) .
$$

Some further results on the oscillation for neutral delay differential equations can be found in the excellent paper of Saker and Kubiaczyk [8] and the recent paper of Ahmed et al. [9]. See also Shen and Debnath [10] and Wang [11]. 
$\mathrm{Li}$ [12] extended results of Chen et al. [7] for (E) in the case when $a(t) \equiv 1$ and introduced some new oscillation criteria under the hypothesis

$$
\int_{t_{0}}^{\infty} q(s) \exp \left\{\frac{1}{\tau} \int_{s}^{\infty} u q(u) d u\right\} d s=\infty .
$$

Kubiaczyk et al. [13] have given some several sufficient conditions for oscillation of all solutions depending on the functions $p$ and $q$ when $p(t)-1$ is allowed to oscillate, while Zhou [14] has established some new sufficient conditions for oscillation depending on an additional constant $\lambda$.

Here, in this paper, we continue in this direction of finding some sufficient conditions for (E) to oscillate in the case when $\int_{t_{0}}^{\infty} q(s) d s<\infty$. To this end, let us site the next two results which will enable us to complete the proofs of our main results.

Lemma 1 (see [8]). Assume that there exists $t^{*} \geq t_{0}>0$ such that

$$
\frac{p\left(t^{*}+n^{*} \tau\right)}{a\left(t^{*}+\left(n^{*}-1\right) \tau\right)} \leq 1 \quad \text { for } n^{*}=0,1,2, \ldots
$$

Let $x(t)$ be an eventually positive solution of $(\mathrm{E})$. Let

$$
z(t)=a(t) x(t)-p(t) x(t-\tau) .
$$

Then

$$
z(t)>0, \quad z^{\prime}(t) \leq 0 .
$$

Theorem 2 (see [15]). Assume that $p(t) \equiv 1$; then every solution of (3) oscillates if

$$
\int_{t_{0}}^{\infty} s q(s) \int_{s}^{\infty} q(u) d u d s=\infty
$$

\section{Main Results}

Theorem 3. Assume that condition (6) holds and either

$$
p(t)+\tau q(t)>0
$$

or

$$
\tau>0
$$

$q(s)$ does not identically equal zero for $s \in[t, t+\tau]$.

Then all solutions of $(\mathrm{E})$ oscillate if and only if the corresponding differential inequality

$$
\begin{aligned}
& (a(t) x(t)-p(t) x(t-\tau))^{\prime} \\
& \quad+q(t) \prod_{i=1}^{n}\left|x\left(t-\sigma_{i}\right)\right|^{\alpha_{i}} \operatorname{sign} x\left(t-\sigma_{i}\right) \leq 0
\end{aligned}
$$

has no eventually positive solution.
Proof. The sufficiency is obvious. To prove necessity, assume that $x(t)$ is an eventually positive solution of (12). We plan to show that (E) has a nonoscillatory solution. Set $z(t)$ as in (7). Then, from (E) we have

$$
z^{\prime}(t)=-q(t) \prod_{i=1}^{n}\left|x\left(t-\sigma_{i}\right)\right|^{\alpha_{i}} \operatorname{sign} x\left(t-\sigma_{i}\right) .
$$

Integrating the last equation from $t$ to $\infty$, and using Lemma 1 , we have

$$
z(t) \geq \int_{t}^{\infty} q(s) \prod_{i=1}^{n} x\left(t-\sigma_{i}\right)^{\alpha_{i}} d s
$$

That is,

$$
a(t) x(t) \geq p(t) x(t-\tau)+\int_{t}^{\infty} q(s) \prod_{i=1}^{n} x\left(t-\sigma_{i}\right)^{\alpha_{i}} d s
$$

which leads to

$$
x(t) \geq \frac{1}{a(t)}\left(p(t) x(t-\tau)+\int_{t}^{\infty} q(s) \prod_{i=1}^{n} x\left(t-\sigma_{i}\right)^{\alpha_{i}} d s\right) .
$$

Let $T>t_{0}$ be fixed so that (16) holds for all $t \geq T$. Set $T_{0}=$ $\max \left\{\tau, \sigma_{i}, 1 \leq i \leq n\right\}$ and consider the set of functions

$$
X=\left\{u \in C\left(\left[T-T_{0}, \infty\right), \mathbb{R}^{+}\right) ; 0 \leq u(t) \leq 1, t \geq T-T_{0}\right\} .
$$

Define a mapping $F$ on $X$ as

$(F u)(t)$

$$
=\left\{\begin{array}{cc}
\frac{1}{a(t) x(t)}(p(t) u(t-\tau) x(t-\tau) & \\
\left.+\int_{t}^{\infty} q(s) \prod_{i=1}^{n} x\left(s-\sigma_{i}\right)^{\alpha_{i}} u\left(s-\sigma_{i}\right)^{\alpha_{i}} d s\right), & t \geq T, \\
\frac{t-T+T_{0}}{T_{0}}(F u)(T)+\left(1-\frac{t-T+T_{0}}{T_{0}}\right), & \\
T-T_{0} \leq t \leq T . &
\end{array}\right.
$$

It is easy to see, by using (16), that $F$ maps $X$ into itself. Moreover, for any $u \in X$ we have $(F u)(t)>0$ for $T-T_{0} \leq t \leq$ $T$.

Next, define the sequence $u_{i}(t)$ in $X$ as follows:

$$
\begin{gathered}
u_{0}(t)=1, \quad t \geq T-T_{0}, \\
u_{j+1}(t)=\left(F u_{j}\right)(t), \quad j=0,1,2, \ldots
\end{gathered}
$$

Therefore, by using (16) and a simple induction, we can easily see that

$$
0 \leq u_{j+1}(t) \leq u_{j}(t) \leq 1, \quad t \geq T-T_{0}, j=0,1,2, \ldots
$$


Set

$$
u(t)=\lim _{j \rightarrow \infty} u_{j}(t) ; \quad t \geq T-T_{0} .
$$

Then from Lebesgue's Dominated Convergence Theorem, it follows that $u(t)$ satisfies

$$
\begin{aligned}
& u(t)= \frac{1}{a(t) x(t)} \\
& \times(p(t) u(t-\tau) x(t-\tau) \\
&\left.\quad+\int_{t}^{\infty} q(s) \prod_{i=1}^{n} x\left(s-\sigma_{i}\right)^{\alpha_{i}} u\left(s-\sigma_{i}\right)^{\alpha_{i}} d s\right), \quad t \geq T, \\
& u(t)= \frac{t-T+T_{0}}{T_{0}}(F u)(T)+\left(1-\frac{t-T+T_{0}}{T_{0}}\right), \\
& T-T_{0} \leq t \leq T .
\end{aligned}
$$

Again set

$$
\omega(t)=u(t) a(t) x(t)
$$

Then

$$
\omega(t)>0, \quad T-T_{0} \leq t<T
$$

and satisfies, for $t \geq T$,

$$
\begin{aligned}
& \omega(t) \\
& =\left[\frac{1}{a(t) x(t)}\right. \\
& \quad \times(p(t) u(t-\tau) x(t-\tau) \\
& \left.\left.\quad+\int_{t}^{\infty} q(s) \prod_{i=1}^{n} x\left(s-\sigma_{i}\right)^{\alpha_{i}} u\left(s-\sigma_{i}\right)^{\alpha_{i}} d s\right)\right] \\
& \quad \times a(t) x(t) .
\end{aligned}
$$

This implies that

$$
\omega(t)=\bar{p}(t) \omega(t-\tau)+\int_{t}^{\infty} \bar{q}(s) \prod_{i=1}^{n} \omega\left(s-\sigma_{i}\right)^{\alpha_{i}} d s, \quad t \geq T,
$$

where

$$
\bar{q}(s)=\frac{q(s)}{\prod_{i=1}^{n} a\left(s-\sigma_{i}\right)^{\alpha_{i}}}, \quad \bar{p}(t)=\frac{p(t)}{a(t-\tau)} .
$$

Clearly, $\omega(t)$ is continuous on $t \geq T-T_{0}$. To show that $\omega(t)$ is positive for all $t \geq T-T_{0}$, assume that there exists $t^{*} \geq T-T_{0}$ such that $\omega(t)>0$ for $T-T_{0} \leq t<t^{*}$ and $\omega\left(t^{*}\right)=0$. Then $t^{*} \geq T$ and by (26) we obtain

$$
0=\omega\left(t^{*}\right)=\bar{p}\left(t^{*}\right) \omega\left(t^{*}-\tau\right)+\int_{t^{*}}^{\infty} \bar{q}(s) \prod_{i=1}^{n} \omega\left(s-\sigma_{i}\right)^{\alpha_{i}} d s
$$$$
t \geq T \text {. }
$$

Then

$$
\bar{p}\left(t^{*}\right) \equiv 0, \quad \bar{q}(s) \prod_{i=1}^{n} \omega^{\alpha_{i}}\left(t-\sigma_{i}\right) \equiv 0, \quad \forall t \geq t^{*} .
$$

This is a contradiction with (10) or (11). Therefore, $\omega\left(t^{*}\right)$ is positive on $\left[T-T_{0}, \infty\right)$. Furthermore, it is easy to see that $\omega(t)$ is a positive solution of $(\mathrm{E})$, which implies that the inequality (12) having no eventually positive solution is a necessary condition for the oscillation of all solutions of (E). The proof is complete.

Remark 4. Theorem 3 is an extent of Theorem 2.1 due to Lalli and Zhang [16], Theorem 1 due to Chen et al. [7], and Theorem 1 due to $\mathrm{Li}[12]$.

Now we give an application of Theorem 3.

Theorem 5. Consider (E) with $n=1$. Suppose that condition (6) holds with

$$
\begin{gathered}
\int_{t_{0}}^{\infty} s \bar{q}(s) \int_{t_{0}}^{s} \bar{q}(u) d u d s=\infty, \\
p(t-\sigma) \bar{q}(t) \geq q(t-\tau),
\end{gathered}
$$

where

$$
\bar{q}(t)=\frac{q(t)}{a(t-\sigma)} .
$$

Then all solutions of $(\mathrm{E})$ are oscillatory.

Proof. Suppose that (E) has an eventually positive solution $x(t)$. Set $z(t)$ as in (7). Then, by Lemma 6 , we have

$$
z(t)>0, \quad z^{\prime}(t) \leq 0 .
$$

From (7), we have

$$
\begin{gathered}
x(t)=\frac{1}{a(t)}(z(t)+p(t) x(t-\tau)), \\
x(t-\sigma)=\frac{1}{a(t-\sigma)}(z(t-\sigma)+p(t-\sigma) x(t-\sigma-\tau)) .
\end{gathered}
$$

Hence, from (E), (31), and (35), respectively, we have

$z^{\prime}(t)=-q(t) x(t-\sigma)$

$$
\begin{aligned}
& =-q(t)\left(\frac{1}{a(t-\sigma)}(z(t-\sigma)+p(t-\sigma) x(t-\sigma-\tau))\right) \\
& =-\frac{q(t)}{a(t-\sigma)} z(t-\sigma)-q(t-\tau) x(t-\sigma-\tau) .
\end{aligned}
$$


That is,

$$
z^{\prime}(t) \leq-\frac{q(t)}{a(t-\sigma)} z(t-\sigma)+z^{\prime}(t-\tau)
$$

or

$$
z^{\prime}(t)-z^{\prime}(t-\tau)+\bar{q}(t) z(t-\sigma) \leq 0
$$

where

$$
\bar{q}(t)=\frac{q(t)}{a(t-\sigma)} .
$$

In view of Theorem 3 , we have that the equation

$$
z^{\prime}(t)-z^{\prime}(t-\tau)+\bar{q}(t) z(t-\sigma)=0
$$

has an eventually positive solution. On the other hand, in view of Theorem 2, condition (30) implies that (40) cannot have an eventually positive solution. This is a contradiction. The proof is complete.

Lemma 6. Suppose that

$$
\begin{gathered}
0<a(t) \leq 1 \\
p(t) \geq 1, \\
\int_{t_{0}}^{\infty} q(s) \exp \left(\frac{1}{\tau} \int_{t_{0}}^{s} u q(u) d u\right) d s=\infty .
\end{gathered}
$$

Let $x(t)$ be an eventually positive solution of $(\mathrm{E})$ and $z(t)$ defined by (7). Then

$$
z(t)<0, \quad z^{\prime}(t) \leq 0 .
$$

Proof. From (E) and (7), we have

$$
z^{\prime}(t)=-q(t) \prod_{i=1}^{n} x\left(t-\sigma_{i}\right)^{\alpha_{i}} \leq 0 .
$$

Therefore, if (44) does not hold, then we have eventually that $z(t)>0$; that is,

$$
a(t) x(t) \geq p(t) x(t-\tau)
$$

which together with (41) and (42) yields

$$
x(t) \geq x(t-\tau) .
$$

Let $t_{1} \geq t_{0}$ be such that

$$
x(t-\tau)>0, \quad \text { for } t_{1} \geq t_{0},
$$

and also such that (16) holds for $t_{1} \geq t_{0}$. Define

$$
\kappa=\min \left\{x(t): t \in\left[t_{1}-\tau, t_{1}\right]\right\} .
$$

Then, $x(t) \geq \kappa$ for $t \geq t_{1}$. Set $\sigma^{*}=\max \left\{\tau, \sigma_{1}, \ldots, \sigma_{n}\right\}$, and we have

$$
x(t) \geq \kappa \quad \text { for } t \geq t_{1}+\sigma^{*}=t_{2} .
$$

For convenience, we denote

$$
N(t)=\left[\frac{t-t_{2}}{\tau}\right]
$$

where $\left[\left(t-t_{2}\right) / \tau\right]$ is the greatest integer parts of $\left(t-t_{2}\right) / \tau$. Then from (7), (41), and (42), we obtain

$$
x(t) \geq a(t) x(t)=z(t)+p(t) x(t-\tau) \geq z(t)+x(t-\tau) .
$$

Thus, we have

$$
\begin{aligned}
x(t) \geq & z(t)+x(t-\tau) \\
\geq & z(t)+z(t-\tau)+\cdots+z(t-(N(t)-1) \tau) \\
& +x(t-N(t) \tau), \quad t \geq t_{2} .
\end{aligned}
$$

But $z(t)$ is decreasing, and $x(t-N(t) \tau) \geq \kappa$ for $t \geq t_{1}$. Therefore, from (53), we get

$$
x(t) \geq N(t) z(t)+\kappa, \quad t \geq t_{1} .
$$

Substituting in (E), we obtain

$$
\begin{array}{r}
z^{\prime}(t)+q(t) \prod_{i=1}^{n}\left[N\left(t-\sigma_{i}\right) z\left(t-\sigma_{i}\right)+\kappa\right]^{\alpha_{i}} \leq 0, \\
t \geq t_{3} \geq t_{2} .
\end{array}
$$

By Holder's inequality, we have

$$
\begin{aligned}
\prod_{i=1}^{n} & {\left[N\left(t-\sigma_{i}\right) z\left(t-\sigma_{i}\right)+\kappa\right]^{\alpha_{i}} } \\
& \geq \prod_{i=1}^{n} N^{\alpha_{i}}\left(t-\sigma_{i}\right) \prod_{i=1}^{n} z^{\alpha_{i}}\left(t-\sigma_{i}\right)+\kappa .
\end{aligned}
$$

Then, from (55), we get

$$
z^{\prime}(t)+q(t) \prod_{i=1}^{n} N^{\alpha_{i}}\left(t-\sigma_{i}\right) z(t)+q(t) \kappa \leq 0, \quad t \geq t_{3} .
$$

Hence,

$$
\begin{aligned}
& {\left[z(t) \exp \left(\int_{t_{3}}^{t} q(s) \prod_{i=1}^{n} N^{\alpha_{i}}\left(s-\sigma_{i}\right) d s\right)\right]^{\prime}} \\
& \quad+\kappa q(t) \exp \left(\int_{t_{3}}^{t} q(s) \prod_{i=1}^{n} N^{\alpha_{i}}\left(s-\sigma_{i}\right) d s\right) \leq 0 .
\end{aligned}
$$

Integrating (58) from $t_{3}$ to $t \geq t_{3}$, we have

$$
\begin{aligned}
& z(t) \exp \left(\int_{t_{3}}^{t} q(s) \prod_{i=1}^{n} N^{\alpha_{i}}\left(s-\sigma_{i}\right) d s\right)-y\left(t_{3}\right)+\kappa \\
& \times \int_{t_{3}}^{t} q(s) \exp \left(\int_{t_{3}}^{s} q(u) \prod_{i=1}^{n} N^{\alpha_{i}}\left(u-\sigma_{i}\right) d u\right) d s \leq 0 .
\end{aligned}
$$


Also we have

$$
\int_{t_{3}}^{\infty} q(s) d s<\infty
$$

Now, by noting that

$$
\left(\frac{\prod_{i=1}^{n} N^{\alpha_{i}}\left(s-\sigma_{i}\right)}{t}\right) \rightarrow \frac{1}{\tau} \text { as } t \longrightarrow \infty,
$$

we see that

$$
\int_{t_{3}}^{\infty} q(s)\left(\frac{s}{\tau}-\prod_{i=1}^{n} N^{\alpha_{i}}\left(s-\sigma_{i}\right)\right) d s<\infty .
$$

Thus, we have eventually that

$$
\lim _{s \rightarrow \infty} \frac{\exp \left(\int_{t_{3}}^{s} q(u) \prod_{i=1}^{n} N^{\alpha_{i}}\left(s-\sigma_{i}\right) d u\right)}{\exp \left((1 / \tau) \int_{t_{3}}^{s} u q(u) d u\right)}
$$

exists. Then, from condition (43), we have

$$
\int_{t_{3}}^{\infty} q(s) \exp \left(\int_{t_{3}}^{s} q(u) \prod_{i=1}^{n} N^{\alpha_{i}}\left(u-\sigma_{i}\right) d u\right) d s=\infty .
$$

Letting $t \rightarrow \infty$ in (59), we obtain a contradiction with (64). The proof is complete.

Remark 7. Lemma 6 extends Lemma 2 in $\operatorname{Li}[12]$ where $a(t) \equiv$ 1.

Theorem 8. Suppose that condition (6) holds with

$$
\begin{gathered}
\int_{t_{0}}^{\infty} \bar{q}(s) \exp \left(\frac{1}{\tau} \int_{t_{0}}^{s} u \bar{q}(u) d u\right) d s=\infty, \\
\prod_{i=1}^{n} \bar{p}_{1}^{\alpha_{i}}\left(t-\sigma_{i}\right) q(t) \geq q(t-\tau),
\end{gathered}
$$

where $\bar{q}$ is defined as in Theorem 3 and

$$
\prod_{i=1}^{n} \bar{p}_{1}^{\alpha_{i}}\left(t-\sigma_{i}\right)=\frac{\prod_{i=1}^{n} p^{\alpha_{i}}\left(t-\sigma_{i}\right)}{\prod_{i=1}^{n} a^{\alpha_{i}}\left(t-\sigma_{i}\right)} .
$$

Then, all solutions of (E) are oscillatory.

Proof. Suppose that (E) has an eventually positive solution $x(t)$. Set $z(t)$ as in (7). Then by Lemma 6 we have

$$
z(t)>0, \quad z^{\prime}(t) \leq 0 .
$$

From (7), we have

$$
\begin{array}{r}
x\left(t-\sigma_{i}\right)=\frac{1}{a\left(t-\sigma_{i}\right)}\left(z\left(t-\sigma_{i}\right)+p\left(t-\sigma_{i}\right) x\left(t-\sigma_{i}-\tau\right)\right) ; \\
i=1,2, \ldots, n .
\end{array}
$$

Hence, from (E) and (69), we have

$z^{\prime}(t)$

$$
\begin{aligned}
& =-q(t) \prod_{i=1}^{n} x^{\alpha_{i}}\left(t-\sigma_{i}\right) \\
& =-q(t) \prod_{i=1}^{n}\left(\frac{1}{a\left(t-\sigma_{i}\right)}\right. \\
& \left.\quad \times\left[z\left(t-\sigma_{i}\right)+p\left(t-\sigma_{i}\right) x\left(t-\sigma_{i}-\tau\right)\right]\right)^{\alpha_{i}} \\
& =-q(t) \prod_{i=1}^{n}\left(\left[\frac{z\left(t-\sigma_{i}\right)}{a\left(t-\sigma_{i}\right)}+\frac{p\left(t-\sigma_{i}\right)}{a\left(t-\sigma_{i}\right)} x\left(t-\sigma_{i}-\tau\right)\right]\right)^{\alpha_{i}} .
\end{aligned}
$$

Applying Holder's inequality, we obtain

$$
\begin{aligned}
z^{\prime}(t) \leq & -q(t) \prod_{i=1}^{n}\left(\frac{z\left(t-\sigma_{i}\right)}{a\left(t-\sigma_{i}\right)}\right)^{\alpha_{i}} \\
& -q(t) \prod_{i=1}^{n}\left(\frac{p\left(t-\sigma_{i}\right)}{a\left(t-\sigma_{i}\right)} x\left(t-\sigma_{i}-\tau\right)\right)^{\alpha_{i}}
\end{aligned}
$$

which implies that

$$
\begin{aligned}
z^{\prime}(t) \leq & -\frac{q(t)}{\prod_{i=1}^{n} a^{\alpha_{i}}\left(t-\sigma_{i}\right)} \prod_{i=1}^{n} z^{\alpha_{i}}\left(t-\sigma_{i}\right)-q(t) \\
& \times \prod_{i=1}^{n}\left[\frac{p\left(t-\sigma_{i}\right)}{a\left(t-\sigma_{i}\right)}\right]^{\alpha_{i}} \prod_{i=1}^{n} x^{\alpha_{i}}\left(t-\sigma_{i}-\tau\right) \\
\leq & -\frac{q(t)}{\prod_{i=1}^{n} a^{\alpha_{i}}\left(t-\sigma_{i}\right)} \prod_{i=1}^{n} z^{\alpha_{i}}\left(t-\sigma_{i}\right)+z^{\prime}(t-\tau),
\end{aligned}
$$

where we have used condition (66) to obtain the last inequality. This implies that $z(t)$ is a positive solution of the inequality

$$
z^{\prime}(t)-z^{\prime}(t-\tau)+\frac{q(t)}{\prod_{i=1}^{n} a^{\alpha_{i}}\left(t-\sigma_{i}\right)} \prod_{i=1}^{n} z^{\alpha_{i}}\left(t-\sigma_{i}\right) \leq 0 ;
$$

that is,

$$
z^{\prime}(t)-z^{\prime}(t-\tau)+\bar{q}(t) \prod_{i=1}^{n} z^{\alpha_{i}}\left(t-\sigma_{i}\right) \leq 0,
$$

where

$$
\bar{q}(t)=\frac{q(t)}{\prod_{i=1}^{n} a^{\alpha_{i}}\left(t-\sigma_{i}\right)} .
$$

As we see, (74) satisfies all conditions of Lemma 6; hence, $Z(t)=z(t)-z(t-\tau)>0$ eventually. On the other hand, since (74) satisfies all conditions of Lemma 6 , then $Z(t)=$ $z(t)-z(t-\tau)<0$ eventually, which is a contradiction. The proof is complete.

Remark 9. Theorem 8 improves and extends Theorem 2 in Chen et al. [7] and Theorem 4 in Li [12], where $a(t) \equiv 1$. See also Theorem 3.4 in $\mathrm{Yu}$ et al. [6]. 


\section{Conflict of Interests}

The authors declare that there is no conflict of interests regarding the publication of this paper.

\section{Acknowledgments}

This research has been completed with the support of the following Grants: FRGS/2/2013/SG04/UKM/02/3 and DIP2012-31.

\section{References}

[1] B. Karpuz and O. Öcalan, "Oscillation criteria for some classes of linear delay differential equations of first-order," Bulletin of the Institute of Mathematics, vol. 3, no. 2, pp. 293-314, 2008.

[2] G. Ladas and Y. G. Sficas, "Oscillations of neutral delay differential equations," Canadian Mathematical Bulletin, vol. 29, no. 4, pp. 438-445, 1986.

[3] I. Kubiaczyk and S. H. Saker, "Oscillation of solutions to neutral delay differential equations," Mathematica Slovaca, vol. 52, no. 3, pp. 343-359, 2002.

[4] F. N. Ahmed, R. R. Ahmad, U. S. Din, and M. S. Noorani, "Oscillation criteria of first order neutral delay differential equations with variable coefficients," Abstract and Applied Analysis, vol. 2013, Article ID 489804, 5 pages, 2013.

[5] Q. Chuanxi, G. Ladas, B. G. Zhang, and T. Zhao, "Sufficient conditions for oscillation and existence of positive solutions," Journal of Applied Analysis, vol. 35, pp. 187-194, 1990.

[6] J. S. Yu, M.-P. Chen, and H. Zhang, "Oscillation and nonoscillation in neutral equations with integrable coefficients," Computers \& Mathematics with Applications, vol. 35, no. 6, pp. 65-71, 1998.

[7] M. P. Chen, J. S. Yu, and L. H. Huang, "Oscillations of first order neutral differential equations with variable coefficients," Journal of Mathematical Analysis and Applications, vol. 185, no. 2, pp. 288-301, 1994.

[8] S. H. Saker and I. Kubiaczyk, "Oscillation of solutions to nonlinear neutral delay differential equations," Journal of Applied Analysis, vol. 8, no. 2, pp. 261-278, 2002.

[9] F. N. Ahmed, R. R. Ahmad, U. K. S. Din, and M. S. M. Noorani, "Some further results on oscillations for neutral delay differential equations with variable coefficients," Abstract and Applied Analysis, vol. 2014, Article ID 579023, 7 pages, 2014.

[10] J. H. Shen and L. Debnath, "Oscillations of solutions of neutral differential equations with positive and negative coefficients," Applied Mathematics Letters, vol. 14, no. 6, pp. 775-781, 2001.

[11] Q. Wang, "Oscillation criteria for first-order neutral differential equations," Applied Mathematics Letters, vol. 15, no. 8, pp. 10251033, 2002.

[12] W. T. Li, "Oscillation of first order neutral nonlinear differential equations with variable coefficients," Indian Journal of Pure and Applied Mathematics, vol. 29, no. 6, pp. 605-615, 1998.

[13] I. Kubiaczyk, S. H. Saker, and J. Morchalo, "New oscillation criteria for first order nonlinear neutral delay differential equations," Applied Mathematics and Computation, vol. 142, no. 2-3, pp. 225-242, 2003.

[14] Y. Zhou, "Oscillation of neutral functional differential equations," Acta Mathematica Hungarica, vol. 86, no. 3, pp. 205-212, 2000 .
[15] J. S. Yu, Z. Wang, and C. X. Qian, "Oscillation of neutral delay differential equations," Bulletin of the Australian Mathematical Society, vol. 45, no. 2, pp. 195-200, 1992.

[16] B. S. Lalli and B. G. Zhang, "Oscillation of first order neutral differential equations," Journal of Applied Analysis, vol. 39, pp. 265-274, 1990. 


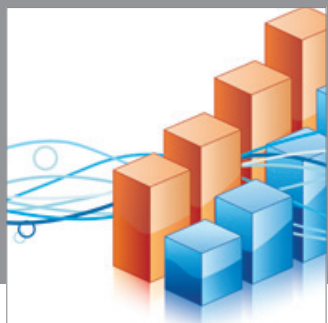

Advances in

Operations Research

mansans

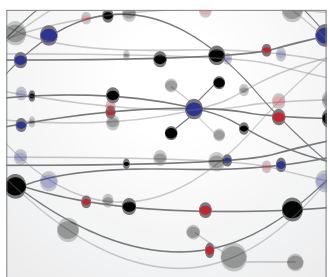

The Scientific World Journal
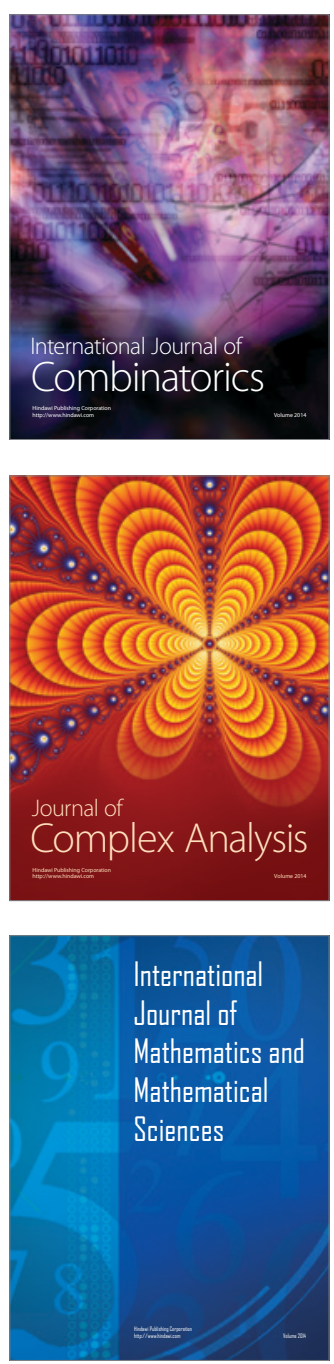
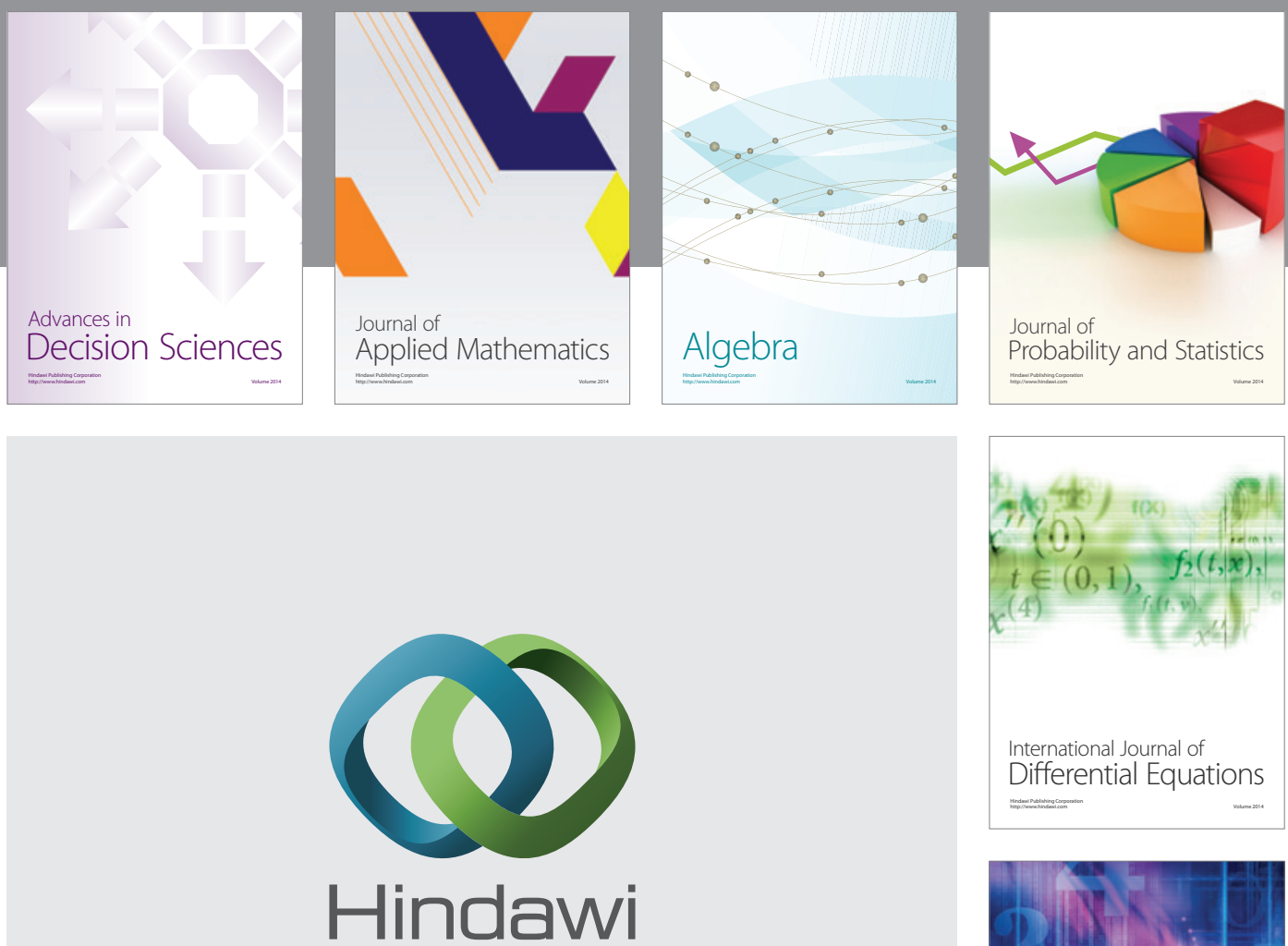

Submit your manuscripts at http://www.hindawi.com
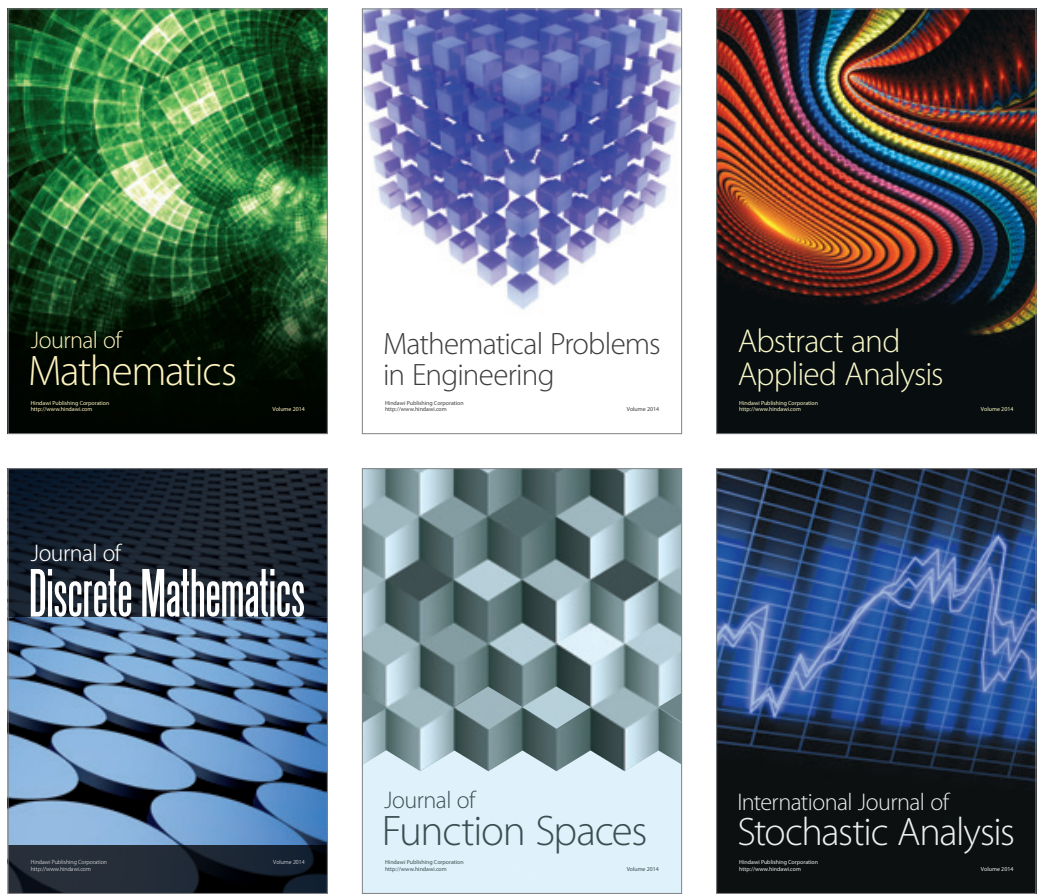

Journal of

Function Spaces

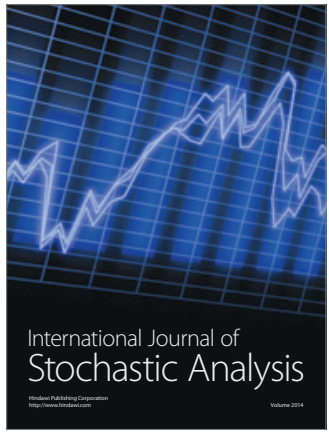

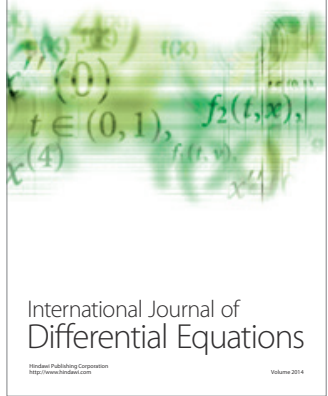
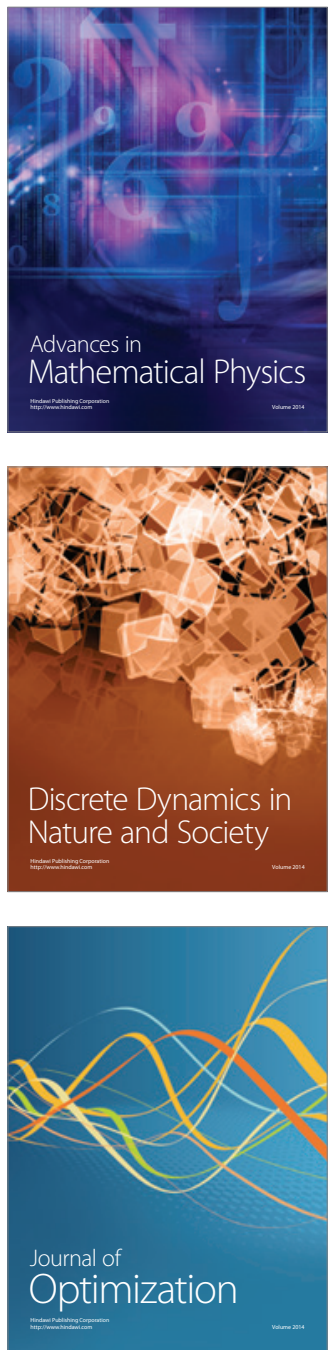